\title{
Keratin 18 phosphorylation as a progression marker of chronic hepatitis $B$
}

Ying Shi ${ }^{1,2 \dagger}$, Shihui Sun ${ }^{1 \dagger}$, Yali Liu ${ }^{2}$, Junfeng $\mathrm{Li}^{1}$, Tong Zhang ${ }^{2}$, Hao Wu ${ }^{2}$, Xinyue Chen ${ }^{2}$, Dexi Chen ${ }^{2 *}$, Yusen Zhou ${ }^{{ }^{*}}$

\begin{abstract}
Background: The intermediate filament proteins keratins 18 (K18) and 8 (K8) polymerize to form the cytoskeletal network in the mature hepatocytes. It has been shown that the phosphorylation of K18 at two serine residues, 33 and 52, correlates with the progression of hepatitis C, but little is known of chronic hepatitis B (CHB). In this study, we examined K18 phosphorylation in relation to CHB.

Results: Site-specific phosphorylation of K18 was determined in livers of twelve healthy donors, and non-cirrhosis $(n=40)$ and cirrhosis $(n=21)$ patients. On average, progressively higher level of Ser52 phosphorylation was observed in non-cirrhotic and cirrhotic livers, while elevated Ser33 phosphorylation was detected in both livers but no significant difference. Progressive increase of Ser33 and Ser52 phosphorylation correlated with the elevation of both histological lesions and enzymatic activities of alanine aminotransferase in non-cirrhotic livers. In the hepatocytes of an inactive HBV carrier, strong signals of Ser33 phosphorylation were co-localized with viral infection, while only basal level of Ser52 phosphorylation was detected in infected cells.
\end{abstract}

Conclusion: Assuming all obtained data, our data suggest that K18 phosphorylation is a progression marker for CHB.

\section{Background}

Keratin 18 (K18) is a member of the intermediate filament family comprising $\sim 70$ cytoskeleton proteins. Adult hepatocytes contain only K18 and keratin 8 (K8), heteropolymerized to form the filament networks that protect the cells from various mechanical stresses [1-3]. Serious hepatocellular injures usually result in damages in the filament scaffolds. For example, during apoptosis K18 is cleaved into small fragments by caspases. As a suitable indicative of hepatocytic apoptosis in vivo, one of the K18 proteolytic fragments, termed tissue polypeptide-specific antigens (TPS), can be readily detected in the plasmas of patients suffering from alcoholic hepatitis [4], nonalcoholic steatohepatitis[5,6], chronic cholecystitis [7] and chronic hepatitis B (CHB) [8]. In addition to scaffolding function, keratin filaments form complex signaling platforms and interact with kinases, adaptors and apoptotic proteins. $\mathrm{K} 18$ is involved in modulating

\footnotetext{
* Correspondence: dexi09@yahoo.com; yszhou@nic.bmi.ac.cn

+ Contributed equally

'State Key Laboratory of Pathogen and Biosecurity, Beijing Institute of Microbiology and Epidemiology, Beijing, China

${ }^{2}$ Department of Infectious Diseases, Capital University of Medical Sciences, Beijing Youan Hospital, Beijing, China
}

hepatocytic apoptosis induced by Fas/TNF family receptors [9-11]. It has been shown that it attenuates TNFinduced cytotoxicity by sequestering the TNF receptor type 1-associated death domain (TRADD) protein from its interaction with the TNF receptor-1 (TNFR1) [12]. K18 is also important for other cellular processes such as mitosis [13], cell cycle progression [14] and responses to stresses [15].

K18 is modified post-translationally at multiple amino acid residues. Many of these modifications are implicated in the functions other than scaffolding. It has been shown that phosphorylation of K18 is most important for its functions in several processes and likely plays a role in liver diseases. For example, the K18 phosphorylation at Ser33 regulates keratin filament organization and modulates its binding to 14-3-3 protein, which in turn, regulates nuclear 14-3-3 redistribution during mitosis and may play a role in hepatocyte mitotic progression $[13,16]$. On the other hand, the phosphorylation at Ser52 could be involved in protecting hepatocytes from toxin- and stress-induced liver injuries [15]. Furthermore, hyperphosphorylation of K18 is associated with the human liver diseases. Site-specific K18 hyperphosphorylation was 
shown to strongly correlate with the progression of liver diseases in patients with chronic noncirrhotic hepatitis $\mathrm{C}$ virus (HCV) [17]. Mallory-Denk bodies (MDBs), hepatic inclusion bodies observed in diverse chronic liver diseases such as alcoholic and non-alcoholic steatohepatitis, chronic cholestasis, metabolic disorders and hepatocellular neoplasms, are composed of aggregates of, in addition to other proteins, $\mathrm{K} 18$ and $\mathrm{K} 8$ in disproportional ratio that are hyperphosphorylated at multiple residues in both keratins, including Ser33 and Ser52 in the former, and Ser73 and Ser 341 in the latter [18-22].

The pathogenesis of viral hepatitis is complex and has been attributed to many factors. Possible mechanisms of chronicity in $\mathrm{HCV}$ include failure of the immune responses to viral infection that results in inappropriate or ineffective induction of cytotoxic $\mathrm{T}$ lymphocytes (CTLs) and production of cytokines. They may lead to continued viral replication, non-specific inflammatory response and fibrosis [23]. Dysregulation of proliferative and apoptotic pathways represents a pro-tumorigenic principle in human hepatocarcinogenesis. Down-regulation of FAS/FAS-L has been detected in patients with chronic hepatitis caused by HCV, suggesting a role of FAS-mediated hepatocytic apoptosis in eliminating infected cells [24]. In the case of primary hepatocellular carcinoma ( $\mathrm{HCC})$, integration of the $\mathrm{X}$ gene $(\mathrm{HBx})$ of hepatitis $\mathrm{B}$ virus (HBV) into the host genome and the ability of the mutant HBx protein to bind to p53 and abrogate p53-mediated apoptosis are implicated in the etiology and molecular pathogenesis $[25,26]$.

The involvement of hepatocytic keratins in apoptosis and other signal pathways prompted us to examine the potential role of $\mathrm{K} 18$ phosphorylation in the progression of HBV chronic hepatitis and/or the severity of liver histological lesions. In this report, we evaluated the phosphorylation of K18 in liver tissues chronically infected with $\mathrm{HBV}$, and determined their enzymatic activities of aspartate (AST) and alanine (ALT) aminotransferases, and histological lesions. Our results indicate that K18 phosphorylation at Ser33 and Ser52 may serve as reliable markers for progression of chronic hepatitis $\mathrm{B}$.

\section{Results}

\section{$\mathrm{K} 18$ phosphorylation is a marker of progression of chronichepatitis B}

HBV infection leads to various clinical presentations, ranging from an inactive carrier state to self-limited acute or chronic hepatitis with progression to cirrhosis and HCC. In this study, we examined the K18 phosphorylation in normal livers and those with chronic non-cirrhotic hepatitis and cirrhosis (Fig. 1A). Liver homogenates containing equal amount of proteins were
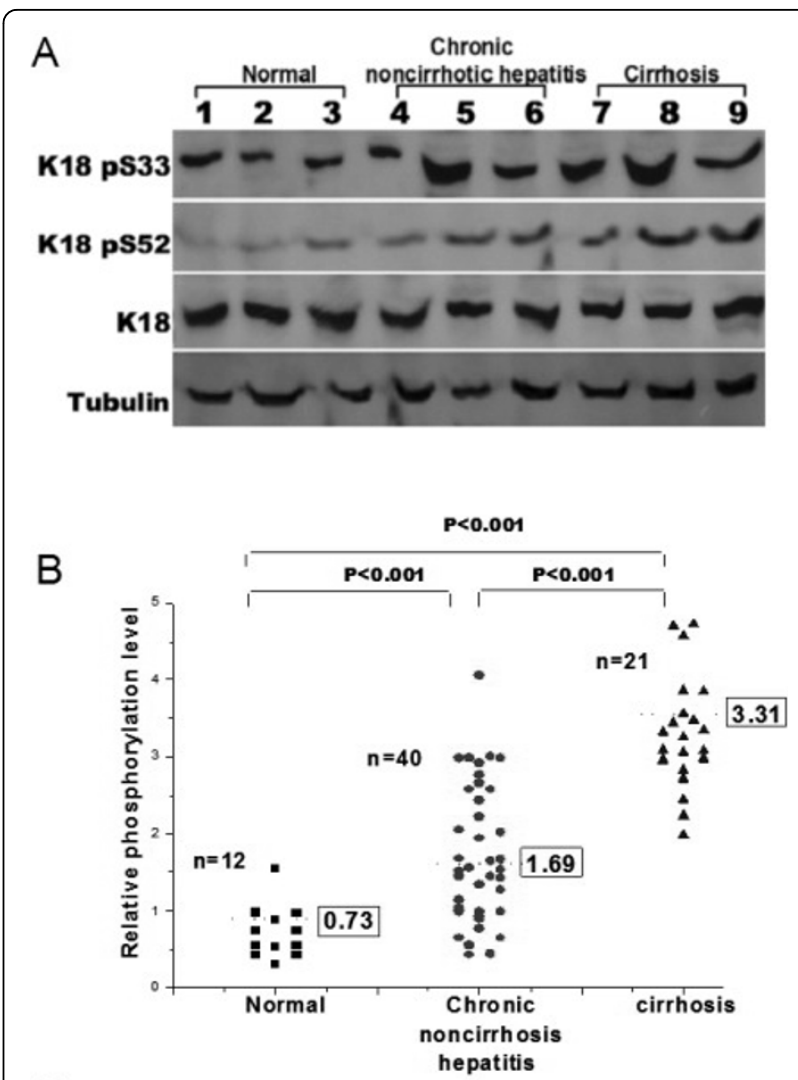

C

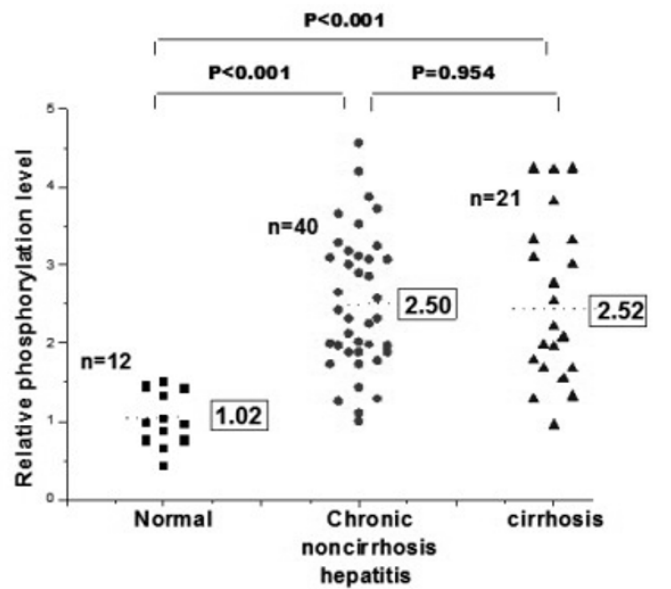

Figure $1 \mathrm{~K} 18$ Phosphorylation in normal, chronic non-cirrhotic hepatitis and cirrhosis livers. (A) Representative immunoblots of tubulin, K18, Ser33 and Ser52 phosphorylated K18 (K18 pSer33 and K18 pSer52, respectively) from livers of indicated sources. (B) Relative levels of Ser33 phosphorylated K18 from livers of healthy donors (squares), and patients with chronic non-cirrhotic hepatitis (circles) and cirrhosis (triangles). The intensity of K18 of each sample was used to normalize that of K18 pSer33, with the average of each group is shown in the box. The P-values of pair-wise comparisons are indicated on the top. (C) Relative levels of Ser52 phosphorylated K18 from livers of indicated sources, with symbols as the same in B. The P-values are indicated on the top. 
analyzed by immunoblots probed with antibodies against phosphorylated serine at specific serine residues in K18, and the relative levels of phosphorylation were quantified. While the levels of Ser52 phosphorylation in the normal livers $(n=12)$ were relatively low with minor fluctuations, those in the chronic non-cirrhotic hepatitis $(n=40)$ varied in a greater range with an average that was significantly higher than the normal controls $(P<$ 0.001) (Fig. 1B). In cirrhotic livers $(\mathrm{n}=21)$, increased levels of Ser52 phosphorylation were detected, with the average significantly higher than the normal $(P<0.001)$ and the chronic non-cirrhotic hepatitis livers $(P<0.001)$ (Fig. 1B). The range of Ser33 phosphorylation in the normal livers was similar to those of Ser52 (Fig. 1C). Significant increases were observed in both chronic non-cirrhotic hepatitis $(P<0.001)$ and cirrhotic $(P<$ $0.001)$ specimens. However, there was no significant difference in the levels of Ser33 phosphorylation between the latter two groups $(P=0.954)$ (Fig. 1C). These results suggest that on average Ser52 phosphorylation increases with the progression of hepatitis B, and that Ser33 phosphorylation increases in hepatitis $B$, but remains largely unchanged with the progression of the disease.

However, much deviation and overlapping of K18 phosphorylation was observed in the two groups of the diseased livers (Figs. 1B and 1C). We then asked the question whether there was any correlation between K18 phosphorylation and subgroups or progression of liver lesions within the same group. The 21 cirrhotic specimens were classified to two groups, active (active $\mathrm{LC}, \mathrm{n}=11$ ) and inactive (inactive $\mathrm{LC}, \mathrm{n}=10$ ) liver cirrhosis (Fig. 2A)[27]. We divided the 41 chronic non-cirrhotic hepatitis livers to three groups according to Ishak's staging classification [28]: minimal histological lesions $(\mathrm{MiH}, \mathrm{n}=13)$, with grading score $<4$ and/or staging score $<2$; medium lesions $(\mathrm{MeH}, \mathrm{n}=17)$, with $4 \leq$ grading scores $<8$ and $2 \leq$ staging score $<3$; and advanced lesions ( $\mathrm{AdH}, \mathrm{n}=10)$, with two scores greater than 8 and 3, respectively (Fig. 2B). The same set of normal livers $(\mathrm{n}=12)$ was included as controls.

The mean relative levels of Ser33 phosphorylation were $1.015,1.710,2.500,3.430,1.790$ and 3.330 for normal, $\mathrm{MiH}, \mathrm{MeH}, \mathrm{AdH}$, inactive LC and active LC subgroups, respectively (Fig. 2D). Significant differences $(P<0.005)$ were observed in any pair of these six subgroups, with the exceptions of $\mathrm{MiH}$ vs. inactive-LC $(P=0.750)$ and AdH vs. active-LC $(P=0.715)$. Similarly, the mean relative levels of Ser52 phosphorylation in the six groups mentioned above were determined to be $0.730,1.078,1.556,2.712,3.003$, and 3.644, respectively (Fig. 2C). Significant differences $(P<0.05)$ were noted in any pair, with the exception of normal vs. $\mathrm{MiH}$ $(P=0.168)$ and AdH vs. inactive-LC $(P=0.289)$. These results indicate an overall increase in K18 phosphorylation at both Ser33 and Ser52 with the progression of CHB. There are however minor differences between these phosphorylation events, as the relative level of Ser33 phosphorylation follows the trend of nor$\mathrm{mal}<\mathrm{MiH} \approx$ inactive $\mathrm{LC}<\mathrm{MeH}<\mathrm{AdH} \approx$ active $\mathrm{LC}$, and that of Ser52 normal $<\mathrm{MiH}<\mathrm{MeH}<\mathrm{AdH}<$ inactive $\mathrm{LC}<$ active $\mathrm{LC}$.

\section{K18 Ser52 phosphorylation is a marker of liver injury, and Ser33 phosphorylation is related to HBV infection}

Many biochemical and serological markers have been used to evaluate liver injuries. Among these, liver functions (e.g., ALT and AST) are most commonly used. We tested $40 \mathrm{CHB}$ livers for their ALT activities and divided them to 3 subgroups: A, ALT $<40 \mathrm{U} / \mathrm{L}$ ( $\mathrm{n}=$ $12) ; \mathrm{B}, 40 \leq \mathrm{ALT}<200 \mathrm{U} / \mathrm{L}(\mathrm{n}=18) ;$ and $\mathrm{C}, \mathrm{ALT}=$ $200 \mathrm{U} / \mathrm{L}(\mathrm{n}=10)$. We then compared K18 phosphorylation in these three groups and the 12 normal livers (Fig. $3 \mathrm{~A})$. Ser52 phosphorylation was equivalent in the control and subgroup A $(P=0.976)$, while significant increase was noted between subgroups $\mathrm{A}$ and $\mathrm{B}(P<$ $0.001)$, and subgroups $B$ and $C(P<0.001)$ (Fig. $3 B)$. The positive correlation between levels of phosphorylation and ALT activities suggests that the phosphorylation of Ser52 in K18 is progressively related to liver injuries. On the other hand, significant increase in Ser33 phosphorylation was detected in group A compared with the control $(P<0.05)$. This increase could be an indicative of HBV infection, in addition to a marker of liver injuries. Incremental increases in Ser33 phosphorylation were also observed between subgroups $\mathrm{A}$ and $\mathrm{B}$ $(P<0.05)$, and $\mathrm{B}$ and $\mathrm{C}(P=0.104)$, respectively.

Since direct comparisons between Ser33 phosphorylation in K18 and HBV infection at a cytomorphological level have not been reported frequently, we sought to determine if there was any correlation. A liver specimen respectively from a randomly selected inactive HBV carrier and a healthy donor was sectioned, and probed for HBsAg and phosphorylated Ser33 or Ser52. In the section of HBV-infected patient maintaining normal liver functions, strong signals of phosphorylated Ser33 were restricted to hepatocytes with strong positive detection of HBsAg (Figs. 4A and 4A'). However, only basal levels of Ser33 phosphorylation were detected in the control (Figs. 4C and 4C'), while basal levels of Ser52 phosphorylation were observed in both HBV positive (Figs. 4B and $4 \mathrm{~B}^{\prime}$ ) and healthy (Figs. $4 \mathrm{D}$ and $4 \mathrm{D}^{\prime}$ ) samples. These results reiterate the conclusion that phosphorylation at Ser33 in K18 is reflective of HBV infection.

\section{Discussion}

Posttranslational modifications, e.g., phosphorylation, glycosylation, acetylation, methylation and ubiquitination, play important roles in functional modulation of 


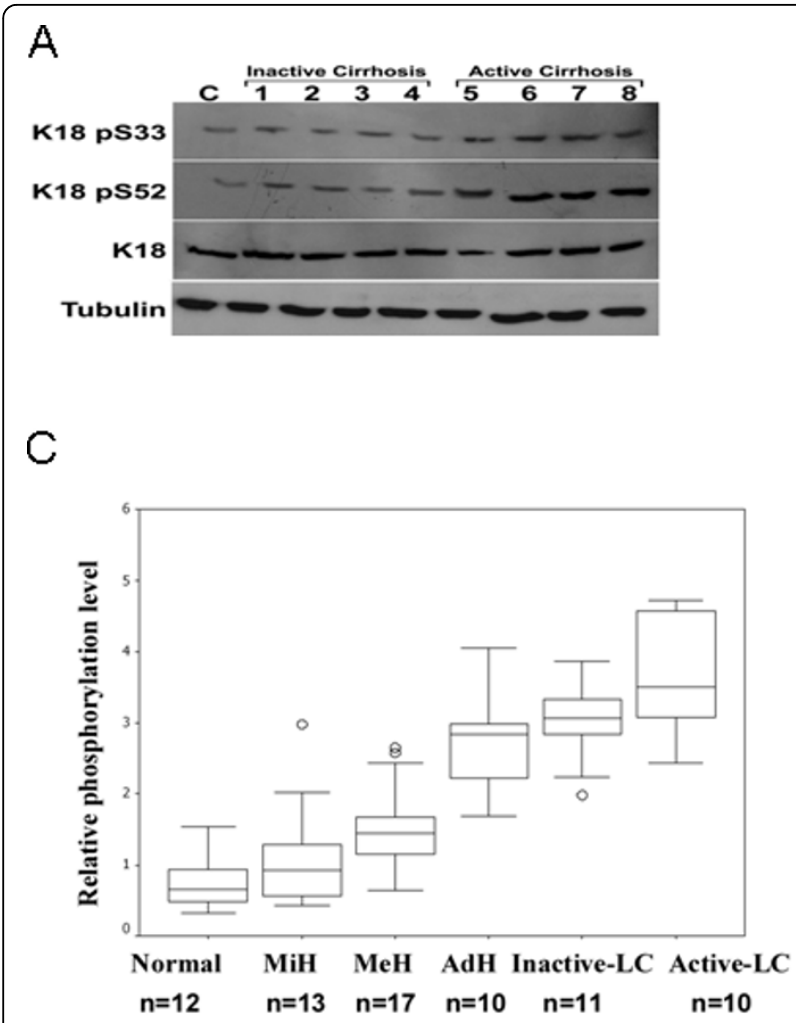

K18 Ser52 phosphorylation in different disease progression stage

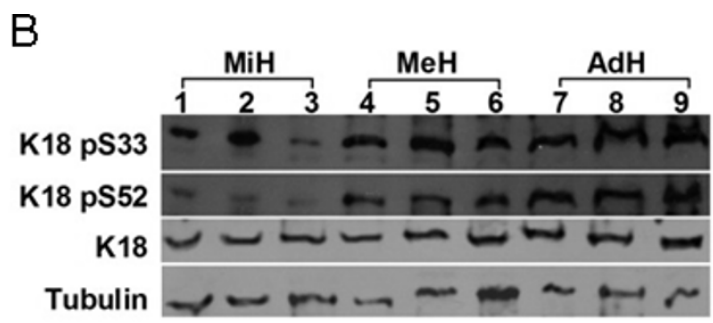

D

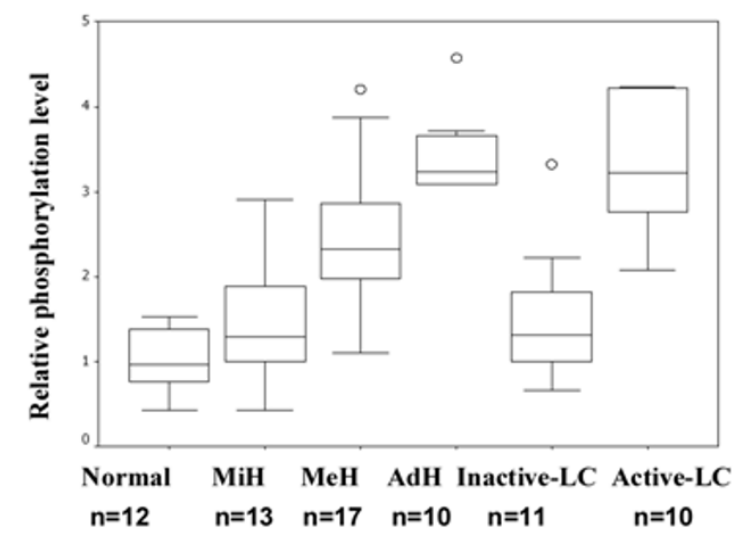

K18 Ser33 phosphorylation in different disease progression stage

Figure 2 K18 phosphorylation in relation to histological progression of CHB. (A) Representative immunoblots of indicated proteins from a control (lane C), inactive (lanes 1-4) and active (lanes 5-8) cirrhotic livers. (B) Representative immunoblots of indicated proteins from livers with progressive histological lesions, MiH (lanes 1-3), MeH (lanes 4-6) and AdH (lanes 7-9), minimal, medium and advanced histological lesions, respectively. (C) The box and whisker plot of the relative levels of Ser33 phosphorylation of K18 in livers of the normal donors, and patients with CHB at different progression stages. Three outliers are indicated by open dots. Significant difference $(P<0.005)$ was observed in any pair with the exception of $\mathrm{MiH}$ vs. inactive LC (liver cirrhosis, $P=0.750$ ), and AdH vs. active LC $(P=0.715)$, respectively. (D) The box whisker plot of relative levels of Ser52 phosphorylation of K18 in livers from indicated sources. The four outliers are indicated by open dots. Significant difference $(P<0.05)$ was observed in any pair with the exception of normal vs. MiH $(P=0.168)$, and AdH vs. inactive $L C(P=0.289)$, respectively.

the intermediate filament proteins. In particular, sitespecific phosphorylation of keratins 8 and 18 is involved in regulating the keratin filament structure, interactions with other proteins and other cellular processes [16,29]. Abnormality and hyperphosphorylation of keratins is associated with a variety of liver injuries and diseases. For example, large aggregates of misfolded hyperphosphorylated $\mathrm{K} 8 / \mathrm{K} 18$, in disproportional ratio, were found in MDBs of alcoholic hepatitis in both humans and mice [18]. The correlation between keratin phosphorylation and hepatitis $C$ led to the suggestion that the former is a progression marker of the latter when increased [17]. It is therefore crucial to investigate whether there is similar correlation between K18 phosphorylation and $\mathrm{CHB}$ caused by viral infection, as it is the most predominant cause of liver disease in China.

We examined liver specimens of 61 patients (40 with chronic non-cirrhotic hepatitis, 21 with cirrhosis) and 12 normal controls. We found that on average Ser52 phosphorylation increased with the progression of $\mathrm{CHB}$, and reached the highest level in cirrhotic livers (Fig. 1C), indicating that it is a progression marker of $\mathrm{CHB}$, as in the case of hepatitis C. Higher levels of Ser33 phosphorylation were also observed in both chronic non-cirrhotic hepatitis and cirrhosis compared with the controls. However, the difference between the two was statistically negligible (Fig. 1B). An earlier study indicated that Ser33 phosphorylation was higher in HCV non-cirrhotic livers than in those of cirrhosis [17]. This discrepancy is likely due to the difference between HBV and HCV hepatitis (see below). These results prompted us to examine the level of K18 phosphorylation in chronic non-cirrhotic livers at different stages as determined by characteristic histological changes, and active and inactive cirrhotic livers (Fig. 2). Although overlapping was observed, Ser52 phosphorylation in general increased progressively with inflammation level, and the average level was higher in active than non-active cirrhosis (Fig. 2D). It is thus 

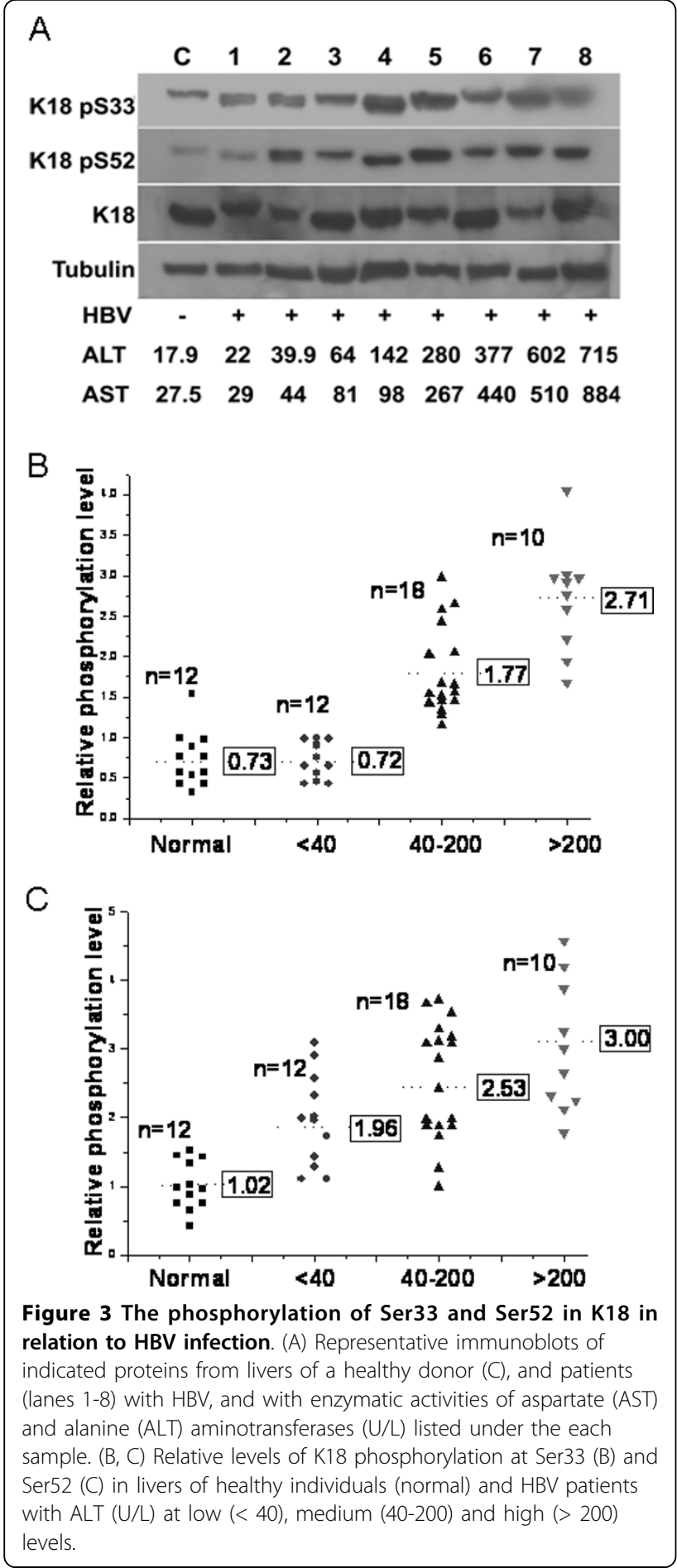

evident that Ser52 phosphorylation reflects largely the chronic progression of liver diseases. Ser33 phosphorylation in the inactive cirrhotic livers, on the other hand, was significantly lower than in the active ones. It was also lower than that in the non-cirrhotic livers with higher levels of inflammation (i.e.; $\mathrm{MeH}$ and $\mathrm{AdH}$ histological subgroups) (Fig. 2C). The level of phosphorylation at Ser33 in K18 seems to correlate with the extent of inflammation. Our results are in consistent with the known implication of Ser33 phosphorylation in resistance to the Fas-mediated apoptosis [10] and sequestering the TRADD protein involved in induction of apoptosis [12], and protective function of Ser52 phosphorylation against hepatotoxic injuries [15].

These conclusions are in agreement with our analyses of the relative level of K18 phosphorylation in livers classified according to ALT activities (Fig. 3). While phosphorylation of Ser52 increased in livers with elevated ALT ( $\geq 40 \mathrm{U} / \mathrm{L}$ ) (Fig. 3C), phosphorylation of Ser33 was observed in HBV infected livers regardless of ALT activities (Fig. 3B). To our knowledge, this is the first time that K18 phosphorylation was directly compared with ALT activities in diseased livers. The association of Ser33 phosphorylation with HBV infection was further demonstrated in the immunohistological analyses of the liver sections of an inactive HBV carrier (Fig. 4). A high level of Ser33 phosphorylation was detected in only hepatocytes infected with $\mathrm{HBV}$, as indicated by the strong HBsAg staining (Fig. 4A and 4A' cf. Fig. 4B and 4B'). On the other hand, we observed merely basal level of Ser52 phosphorylation in the hepatocytes of the carriers regardless of HBsAg staining (Fig. $4 \mathrm{C}$ and $4 \mathrm{C}^{\prime}$ cf. Fig. 4D and 4D'). Toivola and colleagues observed increased phosphorylation of both Ser33 and Ser52 in livers from patients with chronic non-cirrhotic $\mathrm{HCV}$ and cirrhosis [16]. The different cytochemistry and molecular events induced by HBV and HCV could attribute to the difference in Ser52 phosphorylation. Nevertheless, the same authors also observed limited reorganization of the keratin filament networks in pre-cirrhotic HCV livers [17]. The absence of keratin and the destruction of cytoskeletal networks are the hallmarks of hepatocytic injuries [30]. If limited reorganization of the keratin filament networks is also an indication of limited injuries in $\mathrm{HCV}$ livers, the basal level phosphorylation of Ser52 in noncirrhotic HBV livers with relative low ALT activities ( $\leq$ $40 \mathrm{U} / \mathrm{L}$ ) may indicate that in these hepatocytes viral infection does not cause injuries that trigger Ser52 phosphorylation and related cellular protection. If this is indeed true, phosphorylation of Ser33 in K18 could serve as an early indication of HBV infection.

In summary, phosphorylation of Ser33 and Ser52 in K18 may serve as reliable progression markers of chronic hepatitis B. Although it remains to be determined the precise molecular mechanism through which $\mathrm{HBV}$ alters K18 phosphorylation, specifically how differential phosphorylation of Ser33 and Ser52 is modulated in hepatocytes at different stages of the liver disease, our study suggests that the two phosphorylation events may underlie different hepatocytic events associated with HBV. 


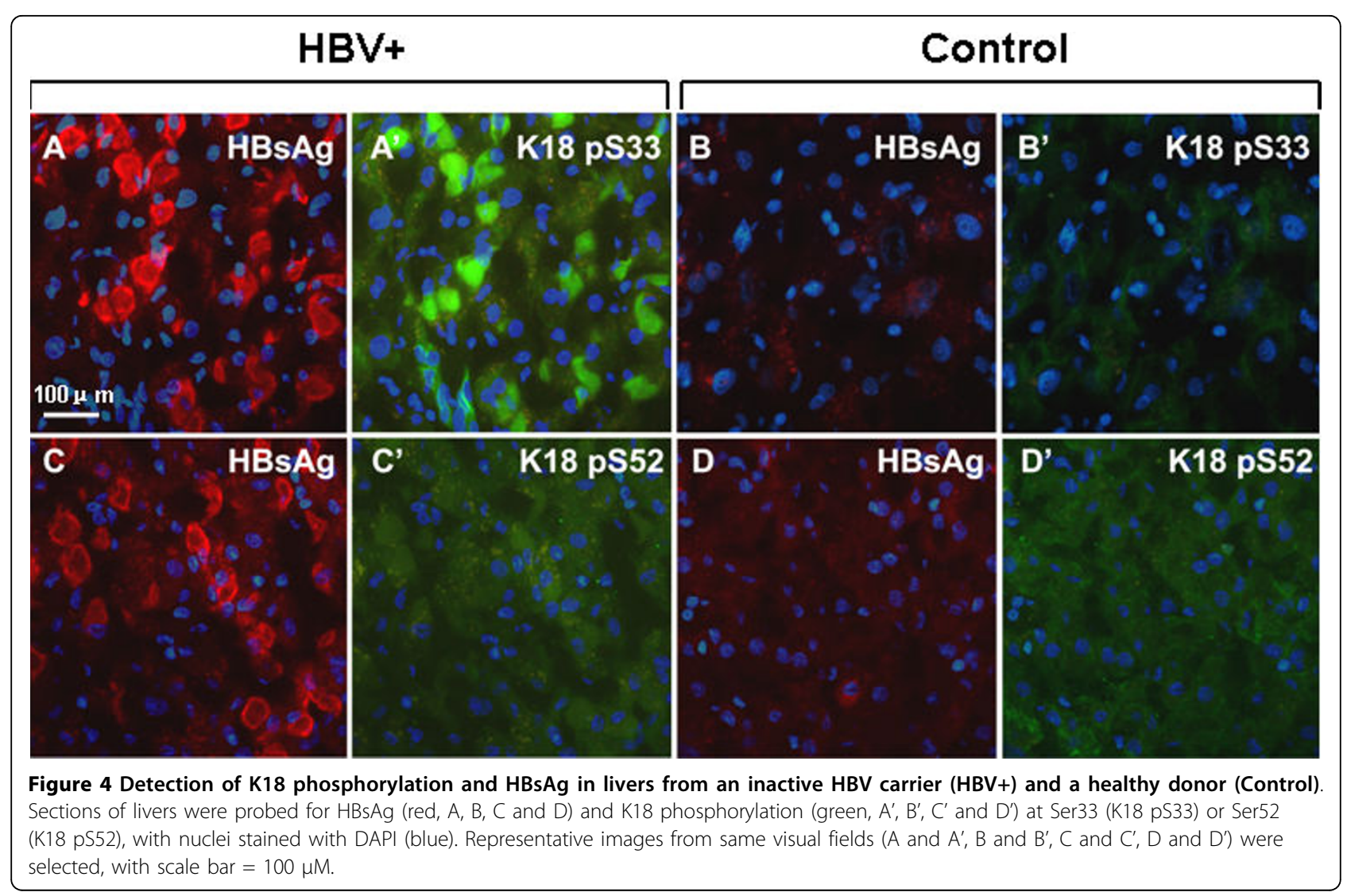

\section{Conclusion}

In conclusion, K18 phosphorylation is a progression marker for CHB. K18 Ser52 phosphorylation is associated with the cellular protection in hepatocytes with HBV infection, while phosphorylation of Ser33 in K18 may serve as an early indication of HBV infection.

\section{Methods}

\section{Antibodies}

The anit-K18 monoclonal antibody and the phosphospecific anti-K18 Ser33 and -Ser52 polyclonal antibodies were from Santa Cruz Biotechnology (Santa Cruz, CA). Anti-HBsAg monoclonal antibody and anti-tubulin monolonal antibody were from Sigma-Aldrich Co (Eugene, OR).

\section{Patients}

Hepatitis B e-antigen (HBeAg)-negative chronic HBV infection was diagnosed based on positive hepatitis $B$ surface antigen (HBsAg) and negative $\mathrm{HBeAg}$ for $\geq 6$ months. Patients with HBeAg-negative chronic HBV infection, persistently normal ALT activity for $\geq 12$ months (determined at least every 3 months during the first year and at least every 6 months thereafter) and baseline serum HBV DNA below 20,000 IU/ml were considered as inactive chronic carriers. ALT and AST were detected by the Hitachi 7600 Series automatic biochemical analyzer (Hitachi, Tokyo, Japan).

A total of $61 \mathrm{HBsAg}$ positive patients were recruited from October, 2006 to September, 2008 at You'an Hospital, Capital Medical University (Beijing, China). They were divided into two groups, 40 patients with $\mathrm{CHB}$ and 21 with liver cirrhosis (LC, active or inactive [27]). Normal control liver tissues were from donors undergone liver transplantation. Those with $\mathrm{CHB}$ were obtained by liver centesis. Cirrhosis specimens were obtained from transplant programs. This study was approved by the local ethics committee, and informed consent was obtained from each patient.

\section{Histological analysis}

Sections of liver biopsy specimens were stained with hematoxylin-eosin, and assessed by a pathologist without prior knowledge of the clinical and virological results. The histological changes were classified according to Ishak et al [28].

\section{Immunoblot analysis}

Liver biopsies or explants were snap-frozen in liquid nitrogen and kept at $-80^{\circ} \mathrm{C}$ until use. The explant pieces 
or liver centesis tissues, of approximately $0.5 \mathrm{~cm}^{3}$, were homogenized in $500 \mu \mathrm{l}$ and $30 \mu \mathrm{l}$, respectively, of high salt lysis buffer $(150 \mathrm{mM} \mathrm{NaCl}, 1 \% \mathrm{NP}-40,0.5 \%$ deoxycholate, $0.1 \%$ SDS, $50 \mathrm{mM}$ Tris [pH 8.0], $5 \mathrm{mM}$ EDTA) with protease inhibitors $(10 \mu \mathrm{g} / \mathrm{ml}$ PMSF). Total cellular lysates were separated on $12 \%$ SDS-PAGE, and then transferred to PVDF membrane. Following the standard protocol, the protein blots was blocked with 5\% nonfat milk, probed sequentially with specific primary antibodies and horseradish peroxidase-conjugated secondary antibodies. The detection of specific proteins on blots was achieved with enhanced chemiluminescence (Pierce SuperSignal, Thermo Fisher Scientific Inc. Rockford, IL) captured on X-ray films. The relevant bands were quantified with a Bio-Rad scanning densitometer (GS-710) using Quantity One software (Bio-Rad Laboratories Inc, version 4.62, Hercules, CA). K18 signal was used to normalize the relative level of phosphorylation of Ser33 and Ser52.

\section{Immunofluorescence microscopy}

Frozen sections of fresh liver tissues were prepared using the standard techniques. They were fixed with $10 \%$ formaldehyde/PBS, incubated in 1\% Triton X-100/ PBS for 10 min, blocked with $3 \%$ BSA/PBS, and probed with mouse anti-HBsAg mAb, for which tetramethyl rhodamine isothiocyanate (TRITC)-conjugated secondary antibodies were used. Slices were then probed with anti-K18 Ser52 or -Ser33 antibodies, for which fluorescent isothiocyanate (FITC)-conjugated second secondary antibodies were used. Nuclei were counterstained with 4,6-diamidino-2-phenylindole (DAPI). Sections were examined with a fluorescence microscope (Nikon Eclipse 80i). Figures were generated with Adobe Photoshop (Adobe Systems Inc, version 7.0, San Jose, CA).

\section{Statistical analysis}

Statistical analyses of the results were performed by Student Newman Keuls Test (SNK) and Least Significant Difference Procedure (LSD) with an analysis software, Statistical Package for Social Science (SPSS Inc. version 11.5, Chicago, IL), and $P<0.05$ was considered statistically significant.

\section{Abbreviations used in this paper}

(HBV): Hepatitis B virus; (CHB): Chronic hepatitis B; (K18): Keratin 18.

\section{Acknowledgements}

This study was supported in part by the National High Technology Research and Development Program of China (863 Program, No. 2006AA02A410), Mega-projects of Science Research for the 11th Five-Year Plan (2008ZX10002-005-3, 2009ZX10004-401) and National Natural Science Foundation of China(30770742).

\section{Author details}

${ }^{1}$ State Key Laboratory of Pathogen and Biosecurity, Beijing Institute of Microbiology and Epidemiology, Beijing, China. ${ }^{2}$ Department of Infectious Diseases, Capital University of Medical Sciences, Beijing Youan Hospital, Beijing, China.

\section{Authors' contributions}

YS was responsible for Western Blotting analysis, interpretation, and writing of this manuscript, SHS was involved in data analysis and drafting the manuscript, YLL carried out immunofluorescence analysis, JFL and TZ coordinated sample collection, HW and XYC revised the manuscript, DXC and YSZ were the principal investigator and were primarily responsible for all aspects of the funding. All authors read and approved the final version.

\section{Competing interests}

The authors declare that they have no competing interests.

Received: 20 January 2010 Accepted: 24 March 2010

Published: 24 March 2010

\section{References}

1. Omary MB, Ku NO, Toivola DM: Keratins, Guardians of the liver. Hepatology 2002, 35:251-257.

2. Coulombe PA, Omary MB: 'Hard' and 'soft' principles defining the structure, function and regulation of keratin intermediate filaments. Curr Opin Cell Biol 2002, 14:110-122.

3. Marceau N, Loranger A, Gilbert S, Daigle N, Champetier S: Keratin mediated resistance to stress and apoptosis in simple epithelial cells in relation to health and disease. Biochem Cel Biol 2001, 79:543-555.

4. Gonzalez-Quintela A, Abdulkader I, Campos J, Fernandez-Hernandez L, Lojo S: Serum levels of keratin-18 fragments [tissue polypeptide-specific antigen (TPS)] are correlated with hepatocyte apoptosis in alcoholic hepatitis. Dig Dis Sci 2009, 54:648-653.

5. Gonzalez-Quintela A, Lojo S, Otero E, Perez LF: Keratin-18 as a marker of steatohepatitis. Hepatology 2006, 44:27-33.

6. Yilmaz Y, Dolar E, Ulukaya E, Akgoz S, Keskin M, Kiyici M, Aker S, Yilmaztepe A, Gurel S, Gulten M, Nak SG: Soluble forms of extracellular cytokeratin 18 may differentiate simple steatosis from nonalcoholic steatohepatitis. World J Gastroenterol 2007, 13:837-844.

7. Simopoulos C, Tsaroucha AK, Asimakopoulos B, Giatromanolaki A, Gavriilidis P, Polychronidis A, Karaiannakis A: Total and caspase-cleaved cytokeratin 18 in chronic cholecystitis: A prospective study. BMC Gastroenterol 2008, 8:14-18.

8. Papatheodoridis GV, Hadziyannis E, Tsochatzis E, Chrysanthos N, Georgiou A, Kafiri G, Manolakopoulos S, Tiniakos DG, Giannousis I, Manesis EK, Archimandritis AJ: Serum apoptotic caspase activity as a marker of severity in $\mathrm{HBeAg-negative} \mathrm{chronic} \mathrm{hepatitis} B$ virus infection. Gut 2008, 57:500-506.

9. Caulin C, Ware CF, Magin TM, Oshima RG: Keratin-dependent, epithelial resistance to tumor necrosis factor-induced apoptosis. J Cell Biol 2000, 149:17-22.

10. Gilbert S, Loranger A, Daigle N, Marceau N: Simple epithelium keratins 8 and 18 provide resistance to Fas-mediated apoptosis. The protection occurs through a receptor-targeting modulation. J Cell Biol 2001, 154:763-773.

11. Ku NO, Soetikno RM, Omary MB: Keratin mutation in transgenic mice predisposes to Fas but not TNF-induced apoptosis and massive liver injury. Hepatology 2003, 37:1006-1014.

12. Inada H, Izawa I, Nishizawa M, Fujita E, Kiyono T, Takahashi T, Momoi T, Inagaki M: Keratin attenuates tumor necrosis factor-induced cytotoxicity through association with TRADD. J Cell Biol 2001, 155:415-426.

13. Ku NO, Michie S, Resurreccion EZ, Broome RL, Omary MB: Keratin binding to 14-3-3 proteins modulates keratin filaments and hepatocyte mitotic progression. Proc Natl Acad Sci USA 2002, 99:4373-4378.

14. Galarneau L, Loranger A, Gilbert S, Marceau N: Keratins modulate hepatic cell adhesion, size and G1/S transition. Exp Cell Res 2007, 313:179-194.

15. Ku NO, Michie SA, Soetikno RM, Resurreccion EZ, Broome RL, Omary MB: Mutation of a major keratin phosphorylation site predisposes to hepatotoxic injury in transgenic mice. J Cell Biol 1998, 143:2023-2032. 
16. Omary MB, Ku NO, Tao GZ, Toivola DM, Liao J: 'Heads and tails' of intermediate filament phosphorylation: multiple sites and functional insights. Trends Biochem Sci 2006, 31:383-394.

17. Toivola DM, Ku NO, Resurreccion EZ, Nelson DR, Wright TL, Omary MB: Keratin 8 and 18 hyperphosphorylation is a marker of progression of human liver disease. Hepatology 2004, 40:459-466.

18. Stumptner C, Omary MB, Fickert P, Denk H, Zatloukal K: Hepatocyte cytokeratins are hyperphosphorylated at multiple sites in human alcoholic hepatitis and in a mallory body mouse model. Am J Pathol 2000, 156:77-90.

19. Denk H, Stumptner C, Zatloukal K: Mallory bodies revisited. J Hepatol 2000, 32:689-702.

20. Zatloukal K, Stumptner C, Fuchsbichler A, Peter F, Carolin L, Michael T, Helmut D: The keratin cytoskeleton in liver diseases. J Pathol 2004, 204:367-376.

21. Fickert P, Trauner M, Fuchsbichler A, Stumptner C, Zatloukal K, Denk H: Mallory body formation in primary biliary cirrhosis is associated with increased amounts and abnormal phosphorylation and ubiquitination of cytokeratins. J Hepatol 2003, 38:387-394.

22. Fausther M, Villeneuve $L$, Cadrin M: Heat shock protein 70 expression, keratin phosphorylation and Mallory body formation in hepatocytes from griseofulvin-intoxicated mice. Comp Hepatol 2004, 3:5-21.

23. Koziel MJ: Cellular immune responses against hepatitis $\mathrm{C}$ virus. Clin Infect Dis 2005, 41(Suppl 1):25-31.

24. Isabel F: Dysregulation of apoptosis in hepatocellular carcinoma cells. World I Gastroenterol 2009, 15:513-520.

25. Schuchmann M, Galle PR: Apoptosis in liver disease. Eur J Gastroenterol Hepatol 2001, 13:785-790.

26. Staib F, Hussain SP, Hofseth LJ, Wang XW, Harris CC: TP53 and liver carcinogenesis. Hum Mutat 2003, 21:201-216.

27. Chinese Medical Association: Infectious and Parasitic Disease, and Hepatology Branches, Prevention and treatment of viral hepatitis. Chin J Hepatol 2000, 8:324-329.

28. Ishak K, Baptista A, Bianchi L, Callea F, De Groote J, Gudat F, Denk H, Desmet V, Korb G, MacSween RN, Phillips MJ, Portmannl BG, Paulsen H, Scheuer PJ, Schmid M, Thaler H: Histological grading and staging of chronic hepatitis. J Hepatol 1995, 22:696-699.

29. Ku NO, Liao J, Chou CF, Omary MB: Implications of intermediate filament protein phosphorylation. Cancer Metastasis Rev 1996, 15:429-444.

30. Bert S, Mieke H, Wendy K, Maartje B, Stephan M, Mathie PGL, Marius N, Viveka B, Peter B, Bertil B, Lane EB, Omary MB, Hans J, Frans CSR: Keratin $8 / 18$ breakdown and reorganization during apoptosis. Exp Cell Res 2004, 297:11-26.

doi:10.1186/1743-422X-7-70

Cite this article as: Shi et al:: Keratin 18 phosphorylation as a

progression marker of chronic hepatitis B. Virology Journal 2010 7:70.

\section{Submit your next manuscript to BioMed Central and take full advantage of:}

- Convenient online submission

- Thorough peer review

- No space constraints or color figure charges

- Immediate publication on acceptance

- Inclusion in PubMed, CAS, Scopus and Google Scholar

- Research which is freely available for redistribution 